\title{
DSP Serial Communication Application Design
}

\author{
Zhengshi Chen ${ }^{(1)}$ \\ Guangzhou Vocational College of Science and \\ Technology, Guangzhou 510550, China, \\ E-mail: E-mail:2636444756@qq.com
}

\author{
Wei Zhang ${ }^{(2)}$ \\ Taiyuan electric power school, Taiyuan 030024, China. \\ E-mail: 20622414091@qq.com
}

\begin{abstract}
This thesis briefly introduces the characteristics of DSP TMS320LF2407A and its asynchronous serial communication interface (SCI), gives a hardware method of SCI and several realizable communication modes are discussed in detail. This design is simple and easy to be operated, and can be used commonly.
\end{abstract}

Key words- DSP; TM320; SCI; Serial Communication

\section{INTRODUCTION}

TMS320LF2407A is produced by TI Company in USA, which is a 16-bit fixed-point DSP mainly designed for digital motor control and other control application systems. It combines high computing function of DSP with strong control ability that faced to motor. Therefore, it becomes ideal substitutes of traditional multimicroprocessor unit and multi-chip design system. Onchip peripherals of TMS320LF2407A contain 16 dualchannel 10-bit A/D converter, clock module with PLL, and WD module with interrupt, SCI and SPI, CAN; in addition, it integrated with two Event Management Module EVA/EVB. Therefore, TMS320LF2407A not only has high-speed data processing ability, but also has control and event managing ability. It can realize manmachine interface, serial communication with host computer, multi-computer communication and so on.

SCI of TMS320LF2407A is a standard and general asynchronous receive/send communication interface. Its receiver and sender are both double buffering, have its own enable and interrupt bit, can work half-duplex and full-duplex. In order to ensure data integrity, SCI does a series of check to receiving data, such as intermittent detection, parity, timeout and frame error. Baud rate of SCI is up to 64kbps. SCI of TMS320LF2407A includes SCIRXD/IO and SCITXD. Registers related with them includes control register and data register. Among these registers, there are 7 control registers. They are respectively used to set data format protocol and communication mode (SCICCR), interrupt enabling and inside clock enabling (SCICTL1), interrupt priority, baud rate (SCIHBAND and SCILBAND) and reflect communication state (SCIXST) and so on. In addition, there are 3 data registers: data-sending buffer register
(SCITXBUF), data-receiving buffer register (SCIRBUF) and simulating data-receiving buffer register (SCIRXEMU).

\section{HARDWARE INTERFACE CIRCUIT OF SERIAL COMMUNICATION}

SCI of TMS320LF2407A supports digital communication between CPU and any other asynchronous peripherals which use standard format. It has two external pins, SCITXD (SCI sending output pin) and SCIRXD (SCI receiving input pin), and signal level of pin is TTL type. But PC serial asynchronous serial communication is based on standard of RS-232, and they are different in signal logical level. Therefore, there is the issue of level translator in the communication between DSP and upper PC. In order to solve the problem, we adopt MAX232 driver IC of MAXIM Company that correspond with RS232 standard. This kind of chip could realize level translator between TTL level and RS-232 interface level. In other words, logical level that $5 \mathrm{~V}$ stands for " 1 " and $0 \mathrm{~V}$ stands for " 0 " could transfer to $-3 \sim-15 \mathrm{~V}$ stands for " 0 " and $+3 \sim+15 \mathrm{~V}$ stands for " 1 ". In addition, communicating accord to RS-232 communication standard, under the promise of communication accuracy, the appropriate distance is no more than 12 meter. So, in order to ensure compatibility and expansibility of hardware design, and could be used in different areas. Another standard communication RS-485 which used MAX485 chip is designed to satisfy remote communication and multicomputer communication requirements. In actual use, through the terminal jumper, it is easy for $\mathrm{J} 3$ and $\mathrm{J} 4$ to achieve the two switch communication standards. System hardware circuit is shown in Figure 1. 


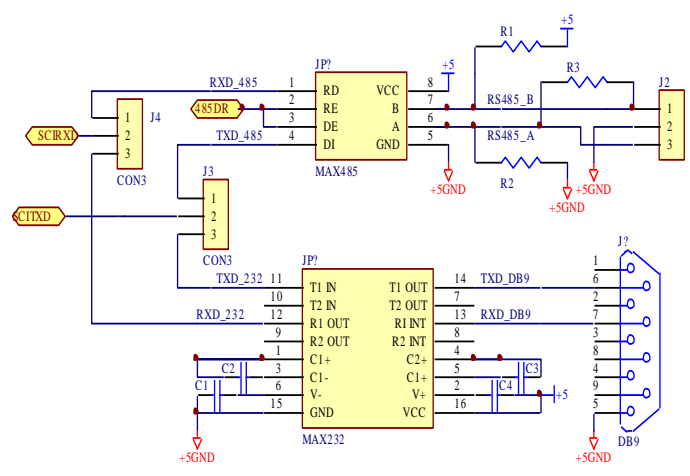

Figure 1. Serial communication hardware circuit

\section{COMMUNICATION METHODS}

Based on the above hardware connection circuit, can achieve the following serial communication methods to meet the needs of different occasions.

\section{A. DSP and Host PC Serial Communication based on the RS-232 protocol}

This communication method is most general way of each kind of control chip communication with the upper pc. Just connect the DSP with the upper pc (DB9) in existing hardware circuit's foundation. The left is choosing appropriate communication protocol and programming communication software. TMS320LF2407A serial communication design has inquires and interrupts two methods to realize the way, usually select the interrupt communication method to save C P U resources. Under the interrupt mode, DSP no longer inquires its status after starting the serial port, but execute according to own master routine order. DSP responds the interrupt again when the serial port has the interrupt request, transfers processes the interrupt communication procedure, and preserves the existing active status including the procedure execution address as well as each control word's condition, and returns to the place which interrupts to continue to carry out own master routine after processing the interrupt routine. Based on the DSP function modulation's characteristic, its serial communication assembly program's mainly divides into the following three steps: (1)Setting clock source module, obtains CPUCLK and SYSCLK (because commutating baudrate with it related); (2)Setting the SCI module, the initialization each SCI control register; (3)Compiling the serial communication interrupt servicing subroutine. Then complete communication between DSP with the PC serial.

\section{B. Point to point communication between DSP}

Because of signal level of TMS320LF2407A is TTL type, so point to point communication does not exist the question of level translator. The simplest way to complete the point-to-point communication between them is shown in Figure 2 that connected corresponding pin directly to complete the hardware connection.

\section{Communication between host PC and multi-DSP that based on $R S-485$ protocol}

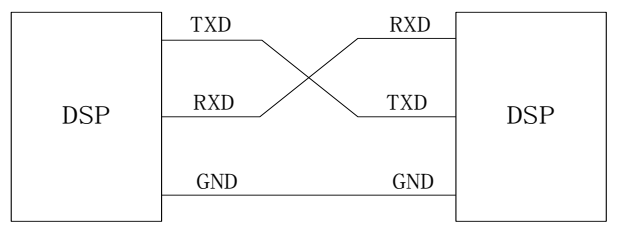

Figure 2. Point to point communication

RS-232 is one of the most popular serial interfaces of PC that used in communication industry. It is defined as single-ended standards that increase communication distance in the low-speed serial communication. RS-232 uses unbalanced transmission, communications distance is limited, generally is only suitable for local communication between devices. RS-485 is different from RS-232, it uses balanced transmission, differentially received data transceiver to drive the bus. It has the characteristic of longer transmission distance, more connection points and lower transmission line cost. Therefore, RS-485 is always used in long distance and multi-computer communication. As circuit shown in Fig. 1, choosing RS-485 mode by jumper terminal J3 and J4, multi-computer communication can be connected as Fig1. Whereas, host PC only has RS-232 interface except RS-485, so a RS232/RS-485 converter is needed to convert interface. In the multi-computer serial communication system that constructed by PC and multi-DSP, master-slave structure is often used: everything is controlled by master PC and slave PC never send orders or data actively. Only one PC can be master PC in multi-computer communication system. Slave PC could not communicate with each other even they need information exchange. The only way is transmitted by master PC.

All multi-computer communication equipments are all linked to the serial bus (TXD and RXD), when host computer communicates with one of the slave computer, how to identify the slave computer and confirm whether the communication with it is multi-computer is a problem that must be solved. The SCI provides two methods: 
multi-computer Idle-line mode and multi-computer Address-bit mode.

- Multi-computer Idle-line mode

In the multi-computer communication, a message frame not only contains the address block but also contains data block and generally data block behind address block. Address information may be constructed by one or more of address blocks and the same of the data information. It also may be constructed by one or more of data blocks. Idle status bits between information blocks that in the same information frame are less than 10; whereas idle status bits between different information frames should be more or equal to 10. Therefore, information frame could be differentiated by the length of idle status bits. User may definite the number of address block and data block by himself.

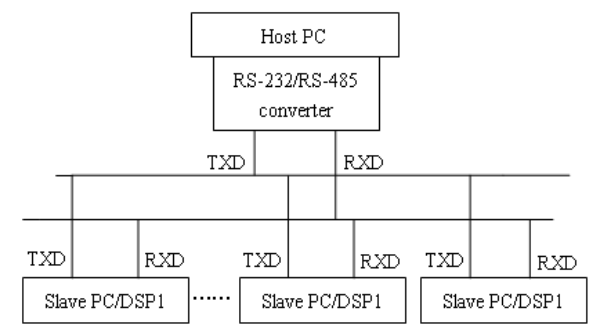

Figuer.3 Multi-computer communication

- Multi-computer address-bit mode

This mode needs some modification in data format, adding an address 1 data bit. It stands for address information if the bit is 1 ; otherwise it stands for data information. Using address-bit mode, it does not need to monitor the length of the idle status and programming is comparably considered. Generally, address-bit mode is suitable for short message transmission, and idle-line information is suitable for long transmission.

\section{COMMUN ICATION SOFTWARE}

In DSP and PC, PC and multi-DSP serial communication system, the upper PC communication program is the key link of the system realization. MSComm (Microsoft Communication Control) is under simplified Windows which Microsoft Corporation provides serial communication programming ActivX controls. It provides two simple methods for the application procedure through the serial interface receiving and dispatching data, moreover in languages and so on VC, VB, Delphi may use. MSComm controlls provides two methods of processing correspondence question: First, the event driven method, mainly uses the OnComm event of MSComm controll to catch and to handle these correspondence event, the OnComm event may also inspect and handle the correspondence mistake. This method's merit is the procedure response is prompt, the reliability is high. Each MSComm controls correspond to a serial port. Another method inquires the way, in this case, whenever after application program execution some serial port operation, will inspect the CommEevent attribute of MSComm control unceasingly to inspect carries out the result or inspects some event whether to occur. Considered timeliness and the reliability, usually select the event driven method to carry on the serial port correspondence programming. The following is uses MSComm control in VB to compile an upper computer correspondence code:

Dim commutime As Integer

//defines the global variable.

Dim Outda (1000) As Integer

Dim Inda (1000) As Integer

Private Sub Form_load()

//serial port initialization

Mscomm1.Comport=1

//serial port 1

Mscomm1.Portopen= True

//open the serial port

Mscomm1.Setting="9600,N,8,1"

//set baudrate, data format

Mscomm1.Inputlen=0

//read all characters

Mscomm1. Inbuffsize $=256$

$/ /$ set receive buffer size

Mscomm1.Outuffsize $=256$

//set transmission buffer size

Mscomm1.Sthreshold=1

//event triggering

Mscomm1.Sthreshold=1

End Sub

Public Sub send-data()

//transmits N data

For $\mathrm{i}=1$ to $\mathrm{N}$

Mscomm1.Output=chr(Outda(i))

Next i

End Sub

Public Sub Mscomm1-Oncomm ()

//event triggering receive data

SelectcaseMscomm1.Commeven

Case ComEvReceive

nda(commutime)=Asc(Mscomm1.input)

$/ /$ put the receive data in the array

commutime $=$ commutime +1

End Select 


\section{End Sub}

In actual application, it also needs some instructions and display controls to display and control system communication, satisfy user request.

\section{CONCLUSIONS}

Through the serial port completing communication between DSP and PC or the DSP, it has simple hardware interface and low cost. Many kinds of communication methods can be chosen to meet different application. This article introduces design method of the TMS320LF2407A chip and the PC serial communication connection has certain commonality and also has a very good reference function to the TMS320F240x series chip.

\section{REFERENCE}

[1] Zewen Wang, Wei Li, Design of the Remote Monitoring System for Mine Hoists, The 24th Chinese control conference proceedings and decisions 2013

[2] Li Zhao-qing, Han Tao. Serial port technology. Beijing: Defense industry Publishing house. 2004.

[3] Based on embedded serial device remote monitoring sys tem[A]. Proceedings 2010 International Conference on I ntelligent Computing and Integrated Systems[C]. 2010
[4] Shuleng Dong,Fang Wang,Jinlan Yu. Serial-Communicati on between PLC and Host Computer in Spatial Carport [A] Proceedings of the 3nd International Conference o n Digital Manufacturing \& Automation(ICDMA2012)[C]. 2012

[5] CAO Lu,WANG Bao-sheng,LI Su-dan,Based on Wireless LAN network performance monitoring and optimization,The 9th China communication society proceedings of academic conference [C]. 2012

[6] Gao Shunkai guo-zhi Yang. Based on the LabVIEW and MCU serial wireless remote control system design [J]. Journal of ship electronic engineering. 2011 (8)

[7] jian-chen zhang. Based on the $\mathrm{c}$ \# serial communication strategy research and implementation of the [J]. Journal of liaocheng university (natural science edition), 2010 (01)

[8] Liang jingjing,Based on RS232 / RS485 temperature monitoring platform development [D]. University of electronic science and technology, (2013.3)

[9] Wang Yanqiu. the human-computer interaction system based on serial communication [D]. The design and implementation of huazhong university of science and technology, (2011.5)

[10] Gao fei, CNC machine tools and production field data collection system research and development [D]. Nanjing university of aeronautics and astronautics, (2012.8) 